\title{
DETERMINACIÓN DE AFLATOXINAS TOTALES (AFs) Y AFB 1 EN DULCES TIPO "MAZAPÁN" DE CACAHUATE Y NUEZ COMERCIALIZADOS EN LA ZONA METROPOLITANA DE GUADALAJARA, JALISCO
}

\section{Determination of total Aflatoxins (AFs) and AFB 1 in sweets type "marzipan" of peanut and walnut marketed in Metropolitan zone of Guadalajara, Jalisco.}

Jiménez-Ortega, Luis A. ${ }^{1}$, Cuevas-Sánchez, Bárbara Y. ${ }^{1}$, Patricio Martínez Severiano ${ }^{1}$, Albarrán Rodríguez Esther ${ }^{2}$, González Zamora Luis F. ${ }^{1}$ y Reyes Velázquez, Waldina $\mathrm{P}^{3 *}$.

${ }^{1}$ Departamento de Salud Pública, ${ }^{2}$ Departamento de Medicina Veterinaria, Centro Universitario de Ciencias Biológicas y Agropecuarias, ${ }^{3}$ Departamento de Ciencias de la Salud y Ecología Humana, Centro Universitario de la Costa Sur, Universidad de Guadalajara. Camino Ramón Padilla N ${ }^{\circ} 2100$. Nextipac, Zapopan, Jalisco, C.P.45110.

*Autor para correspondencia: waldina.reyes@academicos.udg.mx

\section{Resumen}

Las aflatoxinas (AFs) son micotoxinas asociadas con diversos trastornos en la salud humana, destacando efectos de inmunosupresión, mutagénicos, cancerígenos y teratogénicos. La contaminación de alimentos con AFs está ampliamente documentada a nivel mundial, los reportes indican altos niveles en cereales, frutos secos y cacahuate. El propósito del estudio fue detectar la presencia de AFs totales y AFB1 en 66 muestras de dulces tipo "mazapán" de diferentes marcas y lotes, 54 correspondieron al dulce de cacahuate y 12 muestras a los dulces de almendras y nuez. Las técnicas analíticas utilizadas fueron: Cromatografía de inmunoafinidad e Inmunoensayo enzimático (ELISA). Los resultados mostraron contaminación con AFs en el $100 \%$ de las muestras del "mazapán" de cacahuate y nuez, la mayor concentración se observó en el dulce a base de cacahuate $(9.25 \mathrm{mg} / \mathrm{kg})$ diferente estadísticamente $(\mathrm{P}<0.05)$ al nivel promedio del dulce de nuez $(1.17 \mathrm{mg} / \mathrm{kg})$. Los niveles promedio de AFB1 detectados en las muestras de 2 marcas de "mazapán" de cacahuate (niveles promedio: $19.8 \mathrm{mg} / \mathrm{kg}$ $144.17 \mathrm{mg} / \mathrm{kg}$ ) superaron el límite establecido por la legislación internacional para los países exportadores de productos alimenticios a base de cacahuate $(15 \mathrm{mg} / \mathrm{kg})$. En México no existe reglamentación que establezca el límite máximo permitido para AFs y AFB1 en cacahuate, frutos secos y productos derivados por lo que es necesario la revisión de la Normatividad para reducir el riesgo de exposición en la población.

Palabras clave: aflatoxinas, $\mathrm{AFB}_{1}$, mazapán, cacahuate, nuez

Abstract
Aflatoxins (AFs) are mycotoxins associated
with various disorders in human health,
highlighting the effects of mutagenic,
carcinogenic and teratogenic
immunosuppression. The contamination
of food with AFs is documented
worldwide, with reports indicating high
levels in cereals, nuts and peanuts. The
purpose of the study was to detect the
presence of total AFs and AFB1 in 66
samples of "marzipan" type sweets of
different brands and lots. 54
corresponded to peanut sweets and 12


samples to almond and walnut sweets. The analytical techniques used were Immunoaffinity Chromatography and Enzyme Immunoassay (ELISA). The results showed contamination with AFs in $100 \%$ of the peanut and walnut "marzipan" samples. The highest concentration was observed in the peanut-based confectionery $(9.25 \mu \mathrm{g} / \mathrm{kg})$, statistically different $(\mathrm{P}<0.05)$ from the average level of the walnut candy $(1.17 \mu \mathrm{g} /$ $\mathrm{kg}$ ). The average levels of AFB1 detected in the samples of 2 brands of peanut "marzipan" (average levels: $19.8 \mu \mathrm{g} / \mathrm{kg}-144.17 \mu \mathrm{g} / \mathrm{kg}$ ) exceeded the limit established by international legislation for countries exporting peanut-based food products $(15 \mu \mathrm{g}$ $/ \mathrm{kg}$ ). In Mexico, there is no regulation that establishes the maximum limit allowed for AFs and AFB1 in peanuts, nuts and derived products. It is therefore necessary to review the Regulations to reduce the risk of exposure in the population.

Keywords: aflatoxins, AFB1, mazapan, peanut, nut

\section{Introducción}

Las aflatoxinas (AFs) son metabolitos secundarios producidos por hongos filamentosos principalmente de las especies Aspergillus flavus y A. parasiticus. Actualmente se han identificado 18 tipos de AFs, sin embargo, solo aflatoxina $\mathrm{B}_{1}\left(\mathrm{AFB}_{1}\right)$, $\mathrm{AFB}_{2}, \mathrm{AFG}_{1}$ y $\mathrm{AFG}_{2}$ se originan de manera natural en alimentos contaminados por hongos de las especies de Aspergillus aflatoxigénicas. $\mathrm{La} \mathrm{AFB}_{1}$ es la molécula más tóxica dentro de este grupo y es detectada con mayor frecuencia en los alimentos (Bennett y Klich, 2003; Abrar et al., 2013; Roze et al., 2013).

El cuadro clínico generado por la exposición a AFs puede ser de tipo agudo o crónico, el primero se caracteriza por necrosis hemorrágica en hígado (órgano blanco), ictericia, hemorragias gastrointestinales $\mathrm{y}$ puede ocasionar la muerte. La exposición crónica representa el principal impacto a la salud humana, se relaciona con reducción en la absorción de micronutrientes, como la vitamina A y minerales como el selenio y zinc, esenciales para el sistema inmunológico, lo que implica alteración en la modulación de las enfermedades infecciosas, genotoxicidad (formación de aductos con el ADN y con la albumina que induce a la mutación de genes y alteraciones cromosómicas), desarrollo de cáncer en hígado y riñón y teratogénesis al cruzar la placenta humana y generar malformaciones fetales (Williams et al., 2004; Shephard, 2008; Khlangwiset et al., 2011). La Agencia Internacional de Investigaciones contra el Cáncer clasifica a la $\mathrm{AFB}_{1}$ como potencial agente cancerígeno natural en humanos tipo 1(IARC, 2012).

La contaminación de alimentos con AFs depende de numerosos factores, como condiciones ambientales con climas cálidos y húmedos que favorecen la colonización y crecimiento de especies aflatoxigénicas, además la presencia de insectos, el transporte y almacenamiento inadecuado pueden incrementar la producción de dichas micotoxinas (Abrar et al., 2013). Todos los cultivos son susceptibles de la contaminación fúngica, sin embargo, se observa con mayor frecuencia en semillas oleaginosas como cacahuate y en frutos secos como nuez y almendras debido al elevado contenido en carbohidratos, grasas y proteínas que condiciona un medio óptimo para el desarrollo de especies del género Aspegillus (Monyo et al., 2012; Bumbangi et al., 2016). 
Diversos estudios a nivel mundial reportan alta contaminación de AFs en cacahuate, nuez y almendras (Mohammad et al., 2012; Mupunga et al., 2014). La investigación desarrollada en Zimbawe mostró altos niveles de $\mathrm{AFB}_{1}$ en cacahuate, la concentración promedio fue de $51 \mathrm{ng} / \mathrm{g}$ en granos sin procesar y de $75.66 \mathrm{ng} / \mathrm{g}$ en crema de cacahuate, el $100 \%$ de las muestras de la crema de cacahuate excedieron el límite máximo permitido ( $2 \mathrm{ng} / \mathrm{g}$ de $\left.\mathrm{AFB}_{1}\right)$ en dicho país (Mupunga et al., 2014). En México son pocos los estudios realizados sobre la detección de AFs en cacahuate y productos derivados (García-Aguirre y Martínez-Flores, 2006), sin embargo, durante 2004 se reportó el rechazo de 15 lotes de dulce tipo "mazapán" a base de cacahuate de una marca que se elabora en Jalisco, los análisis reportados por el Organismo Internacional para la Administración de Drogas y Alimentos (FDA), indicaron niveles de AFs que superaron el límite máximo permitido en Estados Unidos de Norteamérica $(20 \mu \mathrm{g} / \mathrm{kg})$, el informe se publicó el 19 de abril del mismo año (Mural, 2004).

A nivel mundial las regulaciones sobre los niveles tolerables de aflatoxinas en alimentos se observa en 76 países, estableciendo para AFs totales, niveles de 1 a $20 \mathrm{ng} / \mathrm{g}$ y para $\mathrm{AFB}_{1}$ de 0 a 35 ng/g (FAO, 2004), mientras que la legislación que aplica en 15 países importadores sobre las exportaciones de 31 países permiten adoptar un valor internacional para $\mathrm{AFB}_{1}$ en alimentos de 9 $\mu \mathrm{g} / \mathrm{kg}$ (FAO, 2004), equivalente a la guía del Codex Alimentarius de $15 \mu \mathrm{g} / \mathrm{kg}$ para AFs (CODEX, 2002). La Comisión de regulación Europea es más estricta y considera como límite máximo permitido para $\mathrm{AFB}_{1}$ en cacahuate y frutos secos $2 \mathrm{ng} / \mathrm{g}$ y para AFs totales $4 \mathrm{ng} / \mathrm{g}$ (UE, 2010). En México la regulación sobre AFs en alimentos se presenta en el Diario Oficial de la Federación y se establece en la NOM-247-SSA1-2008, la cual aplica para cereales y derivados, siendo $20 \mu \mathrm{g} / \mathrm{kg}$ el límite máximo permitido para AFs (DOF, 2009), sin incluirse en la Norma otros alimentos como semillas oleaginosas y frutos secos.

En los últimos años se ha incrementado en niños la incidencia de cáncer. Las estadísticas estiman que anualmente se presentan alrededor de 5,000 a 6,000 nuevos casos de cáncer en menores de edad, con un promedio de 2,150 muertes anualmente, siendo la segunda causa de muerte infantil. El $59.2 \%$ de los tumores malignos se presentan en órganos hematopoyéticos, seguido del sistema linfático y tejidos afines (CENSIA/SS, 2015; INEGI, 2017). Debido al alto consumo de dulces tipo "mazapán" elaborados principalmente de cacahuate $\mathrm{y}$ nuez por la población infantil, el objetivo del presente estudio fue analizar la presencia de AFs y $\mathrm{AFB}_{1}$ en diferentes marcas de estos dulces que se comercializan en la zona metropolitana de Guadalajara, ya que la exposición crónica de estas micotoxinas ha demostrado importante riesgo a la salud humana, particularmente en la población infantil.

\section{Materiales y Métodos}

La investigación se realizó durante 2017 en el área de Micotoxicología del laboratorio de Residuos Tóxicos II perteneciente al Departamento de Salud Pública del Centro Universitario de Ciencias Biológicas y Agropecuarias, Universidad de Guadalajara.

Área de estudio y tipo de muestreo:

Se realizó un muestreo puntual en diferentes tiendas de autoservicio y dulcerías ubicadas en la zona metropolitana de 
Guadalajara. El estudio se desarrolló en dos periodos con la finalidad durante el primer muestreo (marzo-mayo) detectar la presencia de AFs totales en dulces "tipo mazapán" a base de almendra, cacahuate y nuez utilizando una técnica de tamizaje rápida (Cromatografía mediante columnas de Inmunoafinidad). El segundo muestreo (junio-julio) se efectuó con el propósito de cuantificar los niveles de $\mathrm{AFB}_{1}$ en el dulce con mayor concentración de AFs, mediante la técnica de ELISA.

Primer muestreo: se analizaron 30 muestras del "mazapán" de tres tipos, 18 muestras fueron de cacahuate (3 marcas comerciales y 3 lotes de c/u), 6 de almendra (3 lotes de una marca) y 6 de nuez (3 lotes de una marca) todas de evaluaron por duplicado. La preparación de las muestras se realizó obteniendo una muestra compuesta de $300 \mathrm{~g}$ de cada marca y lote, por cuarteo se obtuvieron sub-muestras de $50 \mathrm{~g}$ para el análisis mediante la técnica de Cromatografía de inmunoafinidad con detección Fluorométrica. Se utilizaron kits de Aflatest ${ }^{\circledR}$ Vicam Labs con límite de límite de cuantificación de 0.1 a $1000 \mu \mathrm{g} / \mathrm{kg}$.

Segundo muestreo: se analizaron 36 muestras de dulces tipo "mazapán” a base de cacahuate a partir de 6 marcas comerciales y 3 lotes cada una, todas se evaluaron por duplicado. Las muestras se prepararon de manera similar que en la primera etapa y la detección y cuantificación de $\mathrm{AFB}_{1}$ fue mediante la técnica de Inmunoensayo enzimático de tipo competitivo (ELISA), utilizando kits de Agraquant ${ }^{\circledR}$ Romer Labs con límite de cuantificación de 2 a $50 \mu \mathrm{g} / \mathrm{kg}$.

Determinación de aflatoxinas totales (AFs): Técnica de Cromatografía de inmunoafinidad y detección Fluorométrica
Se homogenizaron $50 \mathrm{~g}$ de muestra con 5 g de cloruro de sodio y $100 \mathrm{ml}$ de metanol al $80 \%$ por un minuto en una licuadora comercial a máxima velocidad, para después filtrar a través de papel filtro aflautado (Descripción: 135506 Grade 1289); posteriormente se diluyeron $10 \mathrm{ml}$ del extracto filtrado con $40 \mathrm{ml}$ de agua destilada, procediendo a una segunda filtración en papel microfibra de vidrio (Descripción: 417519 Grade MG 550-HA); $10 \mathrm{ml}$ del extracto filtrado se pasaron a través de la columna de inmunoafinidad, la cual se lavó con dos secuencias de $10 \mathrm{ml}$ de agua destilada. La elución de la micotoxina se efectuó con $1 \mathrm{ml}$ de metanol grado HPLC y se agregó $1 \mathrm{ml}$ del revelador Aflatest. La lectura de la concentración de AFs se efectuó con el Fluorómetro (Series-4 VICAM).

Determinación de AFB 1 Técnica de Inmunoensayo enzimático de tipo competitivo (ELISA)

Se homogenizaron $20 \mathrm{~g}$ de la muestra con $100 \mathrm{ml}$ de metanol al 70\% durante 3 minutos en licuadora comercial a máxima velocidad, posteriormente se filtró en papel Whatman No.1 y se diluyó el extracto filtrado con el buffer del ensayo proporcionado por el fabricante $(100 \mu \mathrm{l}$ del filtrado con $100 \mu \mathrm{L}$ del buffer). Para realizar el inmunoensayo se colocaron los pocillos de dilución y los pocillos con anticuerpos en la microplaca. Se agregaron $200 \mu \mathrm{l}$ del conjugado en cada pocillo, una vez diluido se adicionó $100 \mu \mathrm{l}$ de cada estándar o muestra en el pocillo de dilución el cual contenía $200 \mu \mathrm{l}$ del conjugado. Se mezclaron cuidadosamente con micropipeta 3 veces. Con la pipeta multicanal se transfirieron $100 \mu \mathrm{l}$ del contenido de cada pocillo de dilución al pocillo correspondiente de los anticuerpos. Se incubó durante 15 minutos a temperatura 
ambiente. Posteriormente se vació el contenido de los pocillos en el contenedor de desechos. Se lavaron con agua destilada y descartó el agua de los pocillos. Dicho procedimiento se repitió por 5 ocasiones. Se eliminó el exceso de agua de los pocillos y se agregaron $100 \mu \mathrm{l}$ del substrato en cada pocillo, se procedió a incubar a temperatura ambiente por $5 \mathrm{~min}$. Al término de la incubación se añadió $100 \mu \mathrm{l}$ de la solución Stop en cada pocillo. Se procedió a obtener la densidad óptica en el lector de ELISA (Modelo: ELx 800 de BIO-TEK INSTRUMENTS, INC ${ }^{\circledR}$.) utilizando un filtro de $450 \mathrm{~nm}$ con un filtro diferencial de $630 \mathrm{~nm}$.

\section{Análisis estadístico:}

Los resultados obtenidos fueron contrastados mediante ANOVA a un nivel de significancia de 0.05 . Se compararon los tipos de mazapán y las diferentes marcas de cada uno. El programa estadístico utilizado fue Sigma Stat, versión 3.1.

\section{Resultados y Discusión}

La primera etapa del estudio permitió detectar AFs solo en los dulces tipo "mazapán" a base de cacahuate y nuez ya que el procedimiento de extracción de las muestras utilizado en la técnica de Cromatografía por Inmunoafinidad, no permitió la filtración de las muestras con almendra. Los resultados mostraron contaminación con AFs en el $100 \%$ de las muestras analizadas del "mazapán" de cacahuate y nuez, el Cuadro 1 presenta los niveles promedio por marca y lote de ambos tipos. Las niveles del "mazapán" de cacahuate fueron de 8.1 a $11 \mu \mathrm{g} / \mathrm{kg}$ y en el "mazapán" de nuez de 0.53 a $1.6 \mu \mathrm{g} /$ $\mathrm{kg}$, menores estadísticamente $(\mathrm{p}<0.05)$ a las marcas de cacahuate. Durante la segunda etapa del estudio, se observó contaminación por $\mathrm{AFB}_{1}$ en todas las marcas del "mazapán" de cacahuate, a excepción de 3 lotes analizados (Cuadro 2). Los niveles promedio fueron de $0.67 \mu$ $\mathrm{g} / \mathrm{kg}$ a $144.17 \mu \mathrm{g} / \mathrm{kg}, \quad$ dos marcas mostraron valores significativamente mayores $(\mathrm{P}<0.05)$ al resto de las marcas evaluadas. 
Cuadro 1. Niveles promedio de aflatoxinas totales presentes en dulce tipo "mazapán" de cacahuate y nuez

\begin{tabular}{|c|c|c|c|}
\hline $\begin{array}{l}\text { Tipo de } \\
\text { mazapán }\end{array}$ & $\begin{array}{l}\text { Marca y } \\
\text { No. de lote }\end{array}$ & $\begin{array}{l}\text { Niveles promedio* de } \\
\text { AFs }(\mu \mathrm{g} / \mathrm{kg}) / \text { lote }\end{array}$ & $\begin{array}{l}\text { Promedio } \pm \text { Desviación } \\
\text { estándar de AFs } \\
(\mu \mathrm{g} / \mathrm{kg}) \pm / \mathrm{marca}^{3}\end{array}$ \\
\hline \multirow{11}{*}{ Cacahuate } & $\mathrm{A}-1$ & 8.9 & \multirow{3}{*}{$9.25 \pm 0.67 \mathrm{a}$} \\
\hline & & & \\
\hline & $A-2$ & 8.8 & \\
\hline & $A-3$ & 10.0 & \multirow{5}{*}{$9.13 \pm 0.90 \mathrm{a}$} \\
\hline & $\mathrm{B}-1$ & 8.2 & \\
\hline & & & \\
\hline & $B-2$ & 10.0 & \\
\hline & $\mathrm{B}-3$ & 9.2 & \\
\hline & $\mathrm{C}-1$ & 8.4 & \multirow{3}{*}{$9.16 \pm 1.59 \mathrm{a}$} \\
\hline & & & \\
\hline & $\mathrm{C}-2$ & 8.1 & \\
\hline \multirow{5}{*}{ Nuez } & $\mathrm{C}-3$ & 11 & \multirow{5}{*}{$1.17 \pm 0.57 \mathrm{~b}$} \\
\hline & $\mathrm{D}-1$ & 0.53 & \\
\hline & & & \\
\hline & $\mathrm{D}-2$ & 1.6 & \\
\hline & $\mathrm{D}-3$ & 1.4 & \\
\hline
\end{tabular}

A, B, C= marcas de "mazapán" a base de cacahuate; D= marca de "mazapán" a base de nuez; AFs= aflatoxinas totales. $*=$ El promedio deriva de dos muestras por cada lote y marca. Las literales a y b indican diferencia estadística $\mathrm{P}<0.05$ 
Cuadro 2. Niveles promedio de $\mathrm{AFB}_{1}$ en diferentes marcas y lotes del "mazapán" de cacahuate

\begin{tabular}{cc}
\hline Marcas de Mazapán & $\begin{array}{c}\text { Promedio* }^{*} \pm \begin{array}{c}\text { desviación estándar AFB } \mathbf{~} \mathbf{k g}) \\
(\mathrm{E}-1\end{array} \\
\mathrm{E}-2\end{array}$ \\
$\mathrm{~F}-1$ & $\mathrm{ND}$ \\
$\mathrm{F}-2$ & $2.13 \pm 0.52 \mathrm{~b}$ \\
$\mathrm{G}-1$ & $3.13 \pm 0.19 \mathrm{~b}$ \\
$\mathrm{G}-2$ & $\mathrm{ND}$ \\
$\mathrm{H}-1$ & $0.67 \pm 0.36 \mathrm{~b}$ \\
$\mathrm{H}-2$ & $1.03 \pm 0.18 \mathrm{~b}$ \\
$\mathrm{I}-1$ & $33.62 \pm 3.78 \mathrm{a}$ \\
$\mathrm{I}-2$ & $19.80 \pm 1.13 \mathrm{a}$ \\
$\mathrm{J}-1$ & $1.05 \pm 0.37 \mathrm{~b}$ \\
$\mathrm{~J}-2$ & $1.02 \pm 0.33 \mathrm{~b}$ \\
\hline
\end{tabular}

E, F, G, H, I, J = marcas de "mazapán": 1, 2= lotes ND = No detectado *= El promedio deriva de tres muestras por cada lote y marca. Las literales a y $\mathrm{b}$ indican diferencia estadística $\mathrm{P}<0.05$

Puesto que en México no existe regulación sobre el nivel máximo permitido de AFs en cacahuate, nuez y productos derivados, para analizar los valores detectados en el presente estudio se consideraron los límites establecidos para cacahuate, frutos secos y productos derivados por la Unión Europea (4 $\mu \mathrm{g} / \mathrm{kg}$ ) y por la legislación internacional para países de exportación $(15 \mu \mathrm{g} / \mathrm{kg})$ descrita por la Organización de las Naciones Unidas para la Alimentación y la Agricultura (FAO), observando que los niveles de AFs del "mazapán de cacahuate superaron el límite máximo permitido por la Unión Europea, en tanto, los niveles del dulce de nuez estuvieron dentro de lo establecido por ambos Organismos reguladores (U.E. 2010; FAO,
2004). Respecto a los niveles detectados de $\mathrm{AFB}_{1}$, todas las muestras de una marca del "mazapán" de cacahuate superaron el límite máximo permitido por la FAO $(9 \mu \mathrm{g} / \mathrm{kg})$ en alimentos de exportación (FAO, 2004) y por la Unión Europea $(2 \mu \mathrm{g} / \mathrm{kg})$ para productos a base de cacahuate (U.E. 2010).

La contaminación con aflatoxinas en dulces tipo "mazapán” de cacahuate y nuez, mostraron que el dulce a base de cacahuate presenta niveles de $\mathrm{AFB}_{1}$ de riesgo toxicológico para la población. En México los estudios científicos en alimentos contaminados con AFs se relacionan principalmente al maíz y productos derivados (Plasencia, 2004), así como en leche y 
productos lácteos (Reyes et al., 2018, Landeros et al., 2019). Sin embargo, existen pocos estudios que reportan la detección de AFs en cacahuate $\mathrm{y}$ productos derivados como dulces. Destaca el estudio efectuado en la ciudad de México por García-Aguirre y Martínez-Flores (2006), quienes analizaron 11 muestras de "mazapán" de cacahuate de diferentes marcas con el propósito de establecer el perfil de hongos presentes $y$ cuantificar los niveles de AFs, los resultados mostraron alta incidencia de cepas de Aspergillus flavus, sin detectar contaminación por la micotoxina en ninguna de las muestras evaluadas. Las investigaciones han demostrado con frecuencia el aislamiento de especies de Aspergillus aflatoxigénicas en cacahuate y frutos secos (Monyo et al., 2012; Habibipour et al., 2016), entre los reportes se describe el estudio en la provincia de Hamadan, Irán, donde el análisis de 40 muestras de nuez presentó alta incidencia de especies Aspergillus flavus y A. parasiticus, principales productores de aflatoxinas (Habibipour et al., 2016).

A nivel mundial existen diversos reportes de la contaminación con aflatoxinas en frutos secos y en cacahuate, entre los cuales destaca el estudio realizado en Irán durante 2012, donde fueron analizadas 142 muestras que incluyeron 35 de almendras, 32 de pistaches, 26 de nuez, 13 de avellanas y 6 de cacahuate para cuantificar los niveles de $\mathrm{AFB}_{1}, \mathrm{AFB}_{2}$, $\mathrm{AFG}_{1}$ y $\mathrm{AFG}_{2}$, las determinaciones analíticas fueron realizadas mediante ELISA y HPLC. Los resultados mostraron alto porcentaje de contaminación en los pistaches $(53 \%)$ y en las nueces $(77 \%), \mathrm{AFB}_{1}$ fue la detectada en mayor proporción, los niveles fueron de 9.5 a $43.8 \mu \mathrm{g} / \mathrm{kg}$ en pistaches y de 15.4 a 35.1 $\mu \mathrm{g} / \mathrm{kg}$ en nueces (Mohammad et al., 2012). Posterior estudio desarrollado en la provincia de Hamadan, Irán, el análisis de 40 muestras de nuez mostró mayor contaminación con $\mathrm{AFG}_{1}$ con niveles de 1.7 a $18.2 \mathrm{ng} / \mathrm{g}$, mientras que $\mathrm{AFB}_{1}$ reportó valores de 0 a $8.2 \mathrm{ng} / \mathrm{g}$ (Habibipour et al., 2016). En Malasia, se analizaron 196 muestras de nuez y cacahuate que incluían granos crudos o tostados, además de productos derivados de confitería, los resultados mostraron $16.3 \%$ de muestras positivas a AFs con niveles de 16.6 a 711 $\mu \mathrm{g} / \mathrm{kg}$ (Yin-Hui et al., 2010).

El monitoreo realizado en Taiwan, China durante 14 años (1997 a 2011), mostró contaminación por AFs en cacahuate y productos derivados, se analizaron 1031 muestras de dulces de cacahuate, 142 de crema de cacahuate, 322 de harina de cacahuate, 75 muestras de una bebida a base de arroz y cacahuate y 257 de cacahuate sin procesar. Los resultados reportaron 597 muestras positivas, el mayor porcentaje se observó en la crema de cacahuate (52.8\%), harina de cacahuate $(44.1 \%)$ y en dulces $(32.8$ $\%$ ), los niveles fueron de $0.2 \mu \mathrm{g} / \mathrm{kg}$ a 513.4 $\mu \mathrm{g} / \mathrm{kg}$. Del total muestras analizadas $6.8 \%$ superaron el límite máximo permitido de 15 $\mu \mathrm{g} / \mathrm{kg}$ para AFs (Chen et al., 2013). Posteriormente en otro estudio desarrollado en la misma ciudad se detectó contaminación con AFs en $24 \%$ de las muestras de dulces de cacahuate, el nivel promedio durante 2012 fue de $18.4 \mu \mathrm{g} / \mathrm{kg}$ (rango: $0.3-117 \mu \mathrm{g} / \mathrm{kg}$ ) y en 2013 de $6.0 \mu \mathrm{g} / \mathrm{kg}$ (rango: 0.2-40.5 $\mu \mathrm{g} / \mathrm{kg}$ ) (Chen et al., 2016). Ambos estudios presentaron niveles mayores a los observados en los dulces tipo "mazapán" de cacahuate y nuez del presente estudio.

En Turquía fueron analizadas 112 muestras de dulces tradicionales denominados "walnut sujuk" (a base de nuez) y "Turkish delight" (a base de avellana) para determinar la concentración de $\mathrm{AFB}_{1}$, los 
resultados mostraron $43.8 \%$ de muestras positivas en los dulces de nuez con niveles promedio de $6.1 \mu \mathrm{g} / \mathrm{kg}$ (rango: 0.58 a 152 $\mu \mathrm{g} / \mathrm{kg}$ ) y $60.9 \%$ en los dulces de avellana con niveles de $9.5 \mu \mathrm{g} / \mathrm{kg}$ (rango: 0.43 a 63.4 $\mu \mathrm{g} / \mathrm{kg}$ ), 6 muestras del dulce de nuez y 21 muestras del dulce de avellanas superaron el límite establecido por la regulación de Turquía, de $2 \mu \mathrm{g} / \mathrm{kg}$ y $5 \mu \mathrm{g} / \mathrm{kg}$ para $\mathrm{AFB}_{1}$ respectivamente (Gölge, et al., 2016). En el presente estudio los niveles promedio de $\mathrm{AFB}_{1}$ detectados en 2 marcas del "mazapán" de cacahuate, fueron mayores a los descritos en Turquía.

Entre los países de Latinoamérica destaca el estudio reportado en la ciudad de Rio de Janeiro, Brasil (Lemos Junior et al., 2016), en el cual se analizaron 72 muestras de 36 marcas de dulces denominados "Pacoca" (dulce artesanal indígena a base de cacahuate) de alto consumo en la celebración folclórica anual de 2012, los resultados permitieron observar $37.4 \%$ de muestras positivas a AFs, los niveles promedio fueron de $4.9 \mu \mathrm{g} / \mathrm{kg}$ a $39.6 \mu \mathrm{g} / \mathrm{kg}, 13.8 \%$ de las muestras superaron el límite máximo permitido $(20 \mu \mathrm{g} / \mathrm{kg})$ por la legislación de Brasil para productos de cacahuate (MERCOSUR, 2002; Brasil, 2011).

Debido al impacto de las aflatoxinas sobre la salud humana, se han desarrollado diversos métodos analíticos para la detección y cuantificación de los diferentes tipos de AFs $\left(\mathrm{AFB}_{1}, \mathrm{AFB}_{2}, \mathrm{AFG}_{1}\right.$ y $\left.\mathrm{AFG}_{2}\right)$, entre las principales se mencionan técnicas inmunoquímicas, inmunosensores, inmunoensayos ligados a enzimas y cromatográficos. La cromatografía de líquidos de alta precisión (HPLC), se considera la técnica de elección para la detección y cuantificación de los diferentes tipos de AFs por su sensibilidad, exactitud y reproducibilidad, sin embargo, requiere de técnicos expertos, pretratamiento extenso de las muestras y equipos costosos lo que limita su uso en estudios de monitoreo y tamizaje de gran número de muestras (Espinosa et al., 2011). Entre los métodos alternativos para la detección y cuantificación de $\mathrm{AFs} \mathrm{y}_{\mathrm{AFB}}$ se considera la técnica de ELISA (Ensayo inmunoadsorbente ligado a enzimas), la cual fue aprobada por la AOAC Internacional (Association of Analytical Communities) desde 1990 para los análisis de tamizaje y solo cuando la concentración sistemática de estos procesos es limitada se debe confirmar los valores mediante HPLC. Actualmente es aceptada para publicación en revistas científicas indexadas.

\section{Conclusiones}

Los niveles detectados de $\mathrm{AFB}_{1}$ en el dulce tipo "mazapán” de cacahuate analizados en el presente estudio permiten observar potencial riesgo de exposición a aflatoxinas en la población, principalmente infantil, por lo que es necesario desarrollar nuevas investigaciones en productos derivados del cacahuate como botanas y dulces de alto consumo, además es importante la revisión de la Normatividad en México que controla los límites máximos permitidos para aflatoxinas, ya que solo se establecen valores para AFs totales en cereales y sus productos derivados $(20 \mu \mathrm{g} / \mathrm{kg})$ y para $\mathrm{AFM}_{1}$ en leche y productos lácteos $(0.5 \mu \mathrm{g} / \mathrm{kg})$, metabolito de $\mathrm{AFB}_{1}$ eliminado en la leche de humanos y animales, también asociado con el desarrollo de cáncer. Por otra parte, deben implementarse medida de control y vigilancia en empresas que elaboran productos alimenticios para reducir el riesgo toxicológico en la población. 


\section{Literatura citada}

Abrar, M., Anjum, F.M., Sadiq, B.M., Pasha, I., Atif, M.R., Saeed, F. y Waqas, K. 2013. Aflatoxins: Biosynthesis, Occurrence, Toxicity and Remedies. Critical Reviews in Food Science and Nutrition, $\quad 53$ : $\quad 862-874$, https://doi.10.1080/10408398.2011.5631 54.

Bennett, J.W. y Klich M. 2003. Mycotoxins. Clin. Microbiol Rev, 16(3), 497-516 https://doi.10.1128/CMR.16.3.497516.2003

Brasil. 2011. Ministry of Health. Resolution RDC No.7, de 18 of February of 2011.Disposition of maximum tolerated limits (LMT) from mycotoxins in food.

Bumbangi, N.F., Muma, J.B., Choongo, K., Mukanga, M., Velu, M.R, Veldman, F., Hatloy, A. y Mapatano, M.A. 2016. Occurrence and factors associated with aflatoxin contamination of raw peanuts from Lusaka district's markets, Zambia. Food Control, 68, 291-296 https://doi.org/10.1016/j.foodcont.2016. 04.004

CENSIA/SS. 2015 (Centro Nacional para la Salud de la Infancia y la Adolescencia/Secretaría de Salud). Cáncer infantil en México. http://censia.salud.gob.mx/contenidos/ca ncer/cancer_infantil.html

Chen, Y.C., Liao, C.D., Lin, H.Y., Chiueh, L.C. y Shih, Y.C. D. 2013. Survey of aflatoxin contamination in peanut products in Taiwan from 1997 to 2011. Journal of Food and Drug Analysis, 21, 247-252.

http://dx.doi.org/10.1016//j.jfda.2013.07 $.001 \mathrm{n}$

DOF. 2009. Diario Oficial de la Federación. NOM-247-SSA1-2008. Productos y servicios. Control de aflatoxinas en cereales para consumo humano y animal.
Especificaciones

sanitarias. [Publicación en línea], disponible desde Internet en http://depa.fquim.unam.mx/amyd/archiv ero/NOMcereales_12434.pdf.

Espinosa, A., Contreras-Medina, L.M., Muñoz-Huerta, R.F. y Torres-Pacheco, I. 2011. Cap. 7. Methods for dtection and quantification of Aflatoxins. En: Aflatoxin-Detection, Measurement and Control. InTech. DOI: 10.5772/28439 www.intechopen.com pp. 109-128.

FAO. 2004. Food and Agriculture Organization of the United Nations. Worldwide regulations for mycotoxins in food and feed in 2003. FAO food and nutrition paper 2004; 81. Rome. http://www.fao.org/3/a-y5499s.pdf

García-Aguirre, G. y Martínez-Flores, R. 22006. Mohos y aflatoxinas en mazapán. Revista Mexicana de Micologia, 23, 5052.

http://www.scientiafungorum.org.mx/in dex.php/micologia/article/view/977

Gölge, Ö., Hepsag, F., Kabak, B. 2016. Determination of aflatoxins in walnut sujuk and Turkish delight by HPLC-FLD method. Food Control, 59: 731-736. https://doi.org/10.1016/j.foodcont.2015. 06.035

IARC. 2012. International Agency for Research on Cancer. Aflatoxins. IARC Monographs on the evaluation of carcinogenic risks to humans: chemical agents and related occupations. A review of human carcinogens. Vol. 100 F. International Agency for Research on Cancer, Lyon, France. ISSN 0252-8274. http://publications.iarc.fr/Book-AndReport-Series/Iarc-Monographs-On-The Identification-Of-CarcinogenicHazards-To-Humans/Chemical-AgentsAnd-Related-Occupations-2012

INEGI (Instituto Nacional de Estadística y Geografía) 2017. Estadísticas a propósito del día mundial contra el cáncer (4 de febrero). 
http://www.inegi.org.mx/saladeprensa/a proposito/2017/cancer2017_Nal.pdf.

Khlangwiset, P., Shephard, G,S, y Wu, F. 2011. Aflatoxins and growth impairment: a review. Critical Reviews in Toxicology, 41(9), 740-55. https://doi:10.3109/10408444.2011.575 $\underline{766}$

Landeros, R.P., Noa, P.M., López, L.Y., González, D.G., Noa, E., Real, N. M. Juárez, W.C. y Medina M. 2011. Niveles de Aflatoxina $\mathrm{M} 1$ en leche cruda y pasteurizada comercializada en la zona Metropolitana de Guadalajara, México. Revista de Salud Animal, 34(1), 40-45. scielo.sld.cu/pdf/rsa/v34n1/rsa06112.pdf Lemos, Junior J.W., Trombete, F.M., Reis, L.P.A., Direito, G.M., Randow, A.V. y Saldanha, T. 2016. Aflatoxins intake from peanut candy marketed in Rio de Janeiro city, Brazil. International Food Research Journal, 23: 733-738. https://www.ifrj.upm.edu.my

MERCOSUR. 2002. Reglamento técnico MERCOSUR sobre límites máximos de aflatoxinas admisibles en leche, maní y maíz. GMC/RES. $\quad \mathrm{N}^{\circ}$ 25/02 http://www.mercosur.int/msweb/Norma s/normas_web/Resoluciones/ES/Res_02 5_002_RTM_Aflatoxinas\%20en\%20Le ch-Man\%C3\%ADMa\%C3\%ADz_Acta\%202_02.PDF

Mohammad, R.S.S., Ansarin, M., Tahavori, A., Ghaderi, F. y Nemati, M. 2012. Determination of aflatoxins in nuts of Tabriz confectionariers by ELISA and HPLC methods. Advanced Pharmaceutical Bulletin 2(1), 123-126 https://www.ncbi.nlm.nih.gov/pmc/articl es/PMC3846016/pdf/apb-2-123.pdf

Monyo, E.S., Njoroge, S.M.C., Coe, R., Osiru, M. y Waliyar, F. 2012. Occurrence and distribution of aflatoxin contamination in groundnuts (Arachis hypogaea L) and population density of Aflatoxigenic Aspergilli in Malawi. Crop Protection, 42: 149-155 https://doi.org/10.1016/j.cropro.2012.07. $\underline{004}$

Mupunga, I., Lebelo, S.L., Mngqawa, P., Rheeder, J.P. y Katerere, D.R. 2014. Natural Occurrence of Aflatoxins in Peanuts and Peanut Butter from Bulawayo, Zimbabwe. Journal of Food Protection, 77(10), 18141818 https://doi.org/10.4315/0362028X.JFP-14-129

Mural, $2004 . \quad$ https://muralguadalajara.vlex.com.mx/vid/regresanmazapanes-ve-contaminacion79479609

Njoroge, S.M.C., Matumba, L., Kanenga, K. Moisés Siambi, F.W., Maruwo , J., Machinjiri, N. y Monyo, E.S. 2017. Aflatoxin $B_{1}$ levels in groundnut products from local markets in Zambia. Mycotoxin Research, 33, 113119 https://doi.org/10.1007/s12550-0170270-5

Oliveira, C.A.F., Gonçalves, N.B., Rosim, R.E. y Fernandes, A.B. 2009. Determination of aflatoxins in Peanut Products in the Northeast Region of São Paulo, Brazil. International Journal of Molecular Sciences 10(1): 174-183. https://doi.org/10.3390/ijms10010174

Plasencia, J. 2004. Aflatoxins in Maize: A Mexican Perspective, Journal of Toxicology: Toxin Reviews, 23:2-3, 155-177 https://doi.org/10.1081/TXR200027809

Reyes-Velázquez W.P., Palomera, G.C., Patricio, M.S., Toral F.A., Landeros, R.P., Noa, P.M. y Rojo, F. 2018. Contaminación con Aflatoxina M1 en leche y quesos en México con análisis retrospectivo mundial 2008-2017. Memorias. XX Congreso Internacional de Inocuidad de Alimentos. Celebrado en Nuevo Vallarta, Nayarit del 1 al 3 de noviembre de 2018.

Roze, L.V., Hong, S.Y. y Linz, J.E. 2013. Aflatoxin Biosynthesis: Current 
Frontiers. Annual Review of Food Science and Technology 4(1), 293-311 https://doi: 10.1146 / annurev-food$\underline{083012-123702}$

Shephard, G.S. 2008. Impact of mycotoxins on human health in developing countries Food Additives and Contaminants, 25(2), 146-151 Parte A, DOI:10.1080 / 02652030701567442

UE. 2010. Reglamento (CE) No 178/2010 de la Comisión de 2 de marzo de 2010 por el que se modifica el Reglamento (CE) $n^{\circ}$ $401 / 2006$ en lo que respecta a los cacahuates y otras semillas oleaginosas, a los frutos de cáscara arbóreos, a los huesos de albaricoque, al regaliz y al aceite vegetal. DO L 52 de 3 de marzo de 2010, p. 32-34

Williams, J.H., Phillips, T.D., Jolly, P.E., Stiles, J.K., C.M. Jolly, y Aggarwal, D. 2004. Human Aflatoxicosis in developing countries: a review of toxicology, exposure, potential health consequences, and interventions. The American Journal of Clinical Nutrition, 80(5), 1106- 1122. https://doi.org/10.1093/ajen/80.5.1106

Yin-Hui, L., Noryati, I., Aishah, A.L. y Rosma, A. 2010. Aflatoxin occurrence in nuts commercial nutty products in Malaysia. Food Control 21(3), 334-338. https://doi.org/10.1016/j.foodcont.2009. $\underline{06.002}$. 